\title{
Lucha hegemónica y élites políticas: rearticulaciones de fuerzas y desafios al proceso de cambio en Bolivia y Venezuela
}

\section{Hegemonic struggle and political elite: rearticulation of forces and challenges to the 'proceso de cambio' in Bolivia and Venezuela}

\section{DOI:10.5752/P.2317-773X.2017v5.n3.p90}

Mayra Goulart ${ }^{1}$

Ana Carolina Teixeira Delgado ${ }^{2}$

e Política Internacional na Universidade Federal Rural do Rio de Janeiro (UFRRJ). ORCID:

2. Professora del Centro Interdisciplinar de Integração e Relações Internacionais da Universidade Federal da Integração Latino-A mericana (Unila). Ex-Pesquisadora pós-doc do Programa de Pós-Graduação em Relações Internacionais (PPGRI) da UERJ. Doutora e mestre em Relações Internacionais pela Pontifícia Universidade Católica do

Rio de Janeiro (PUC-Rio), com estágio doutoral no CIDES-UMSA. Possui graduação em Ciências Sociais pela Universidade Federal do Rio de Janeiro (UFRJ). Foi assistente de coordenação do Observatório Político Sul-Americano (Opsa), vinculado ao Instituto de Estudos Sociais e Políticos da Universidade do Estado do Rio de Janeiro (lesp-Uerj), e assistente de pesquisa no BRICS Policy Center. Foi professora na graduação de

Relações Internacionais da PUC-Rio, onde também foi Coordenadora Adjunta, e da UnilaSalle-Niterói. Suas pesquisas possuem caráter multidisciplinar, com ênfase nos seguintes temas: descolonização, globalização, movimentos sociais, gênero, meio ambiente e América Latina. ORCID: 0000-0002-6955-1586.
Recebido em: 16/06/2017

Aceito em: 21/02/2018

\section{RESUMÉN}

El inicio del siglo XXI en Sudamérica se caracterizó por la emergencia de gobiernos "progresistas". En Venezuela y Bolivia, estos gobiernos han logrado obtener mayorías sucesivas en el Congreso, impulsando a los "procesos de cambio" pero reduciendo la representación de grupos de interés minoritarios. En este artículo nuestro objetivo es contrastar las experiencias venezolana y boliviana, poniendo en relieve la reconfiguración de sus élites y fuerzas políticosociales que los condicionaron. Como hipótesis central, argumentamos que la polarización entre gobierno y oposición, crucial en los dos casos, asume características distintas respecto a los grados de pluralidad interna de ambos bloques.

Palabras clave: Proceso de cambio. Bolivia. Venezuela. Élites políticas. Fuerzas político-sociales. Congreso.

\section{ABSTRACT}

the beginning of the 21st century in South America was marked by the emergence of "progressive" governments. In Venezuela and Bolivia, those governments achieved majority in Congress successively, prompting the proceso de cambio in each country but reducing the representation of minority groups of interest. In this paper our goal is to contrast Venezuelan and Bolivian experiences, especially regarding the reconfiguration of elite and social-political forces that influenced such processes. As central hypothesis, we argue that polarization between government and opposition, although crucial in these two cases, assumes different characteristics according to the degree of internal plurality of both blocks.

Keywords: Change process. Bolivia. Venezuela. Political elites. Social-political forces. Congress. 
Introducción

El inicio del siglo XXI representa un período de reordenamiento de la escena política sudamericana paralelamente a la reconfiguración de poder observada en el ámbito internacional. De un lado, la presencia de las llamadas potencias emergentes en las instituciones internacionales y sus crecientes demandas por commodities, en especial China, han contribuido para una descentralización del poder y el énfasis de las economías de Sudamérica en la extracción y exportación de sus recursos naturales. En ese sentido, estos cambios han promocionado lo que muchos teóricos coincidieron identificar como un modelo desarrollista-extractivista, marcado por la reprimarización de las actividades económicas y el recrudecimiento de las tensiones sociales (ACOSTA, 2011; GUDYNAS, 2009; SVAMPA, 2013; VELTMEYER; PETRAS, 2014). De otro lado, la llegada al poder de gobiernos "progresistas", cuyos proyectos buscaron incluir en alguna medida las reivindicaciones de grupos relegados al margen de los procesos políticos nacionales, reflejó un momento de mudanza de imagen en la región. En Venezuela y Bolivia, estos hechos se tradujeron en la expresión "proceso de cambio", que condensa los intentos de refundación del Estado, vinculados a la creación de nuevas cartas constitucionales, sus dispositivos de empoderamiento de la sociedad civil, y la adopción de un modelo económico centrado en el ente estatal como promotor del desarrollo. También en estos países, las transformaciones en el panorama nacional resultaron en la recomposición de la arena política, desplazándose a las antiguas élites dominantes en la medida que nuevos actores se establecían en el poder.

En ambos los casos, los gobiernos han logrado obtener amplias mayorías en el Poder Legislativo, permitiendo la aprobación de sus proyectos y reduciendo, en mayor o menor grado, la importancia de los procesos de negociación entre los diferentes grupos parlamentarios. Esta situación se observa principalmente en Venezuela, donde el éxito electoral del chavismo desde 1999 puede ser apuntado como el principal instrumento del oficialismo en la lucha hegemónica trabada en el país. Sin embargo, también son muchas las diferencias entre las dos experiencias: contextuales (empezaron en momentos y en sociedades distintas), temáticas (en Bolivia, sobresalen la descolonización, las autonomías, mientras en Venezuela la disputa ocurre alrededor del socialismo), con relación al grado de pluralidad de las élites políticas.

En este artículo, nuestro objetivo es contrastar las experiencias venezolana y boliviana, poniendo en relieve los desdoblamientos de las mismas, sobre todo con relación a la reconfiguración de sus élites bien como de las fuerzas político-sociales que condicionan los respectivos procesos. Para eso, nos anclaremos en los conceptos-clave de hegemonía y populismo. Como hipótesis central, argumentamos que la polarización entre gobierno y oposición, aunque crucial en los dos casos, asume características distintas respecto a los grados de pluralidad interna de ambos bloques y las particularidades de los respectivos procesos políticos. En ese sentido, Bolivia se destaca por la diversidad de actores de matices ideológicos variados y su división actual: de una parte, estos grupos se aglutinan alrede- 
dor de la órbita gubernamental a partir de una retórica descolonizadora e indigenista, mientras antiguos aliados y algunos liderazgos indígenas se convierten en oposición. Este proceso tiene como telón de fondo la expansión del Movimiento al Socialismo (MAS), constituyéndose como el más grande partido político del país, y la desarticulación de las élites oligárquicas (cruceña, especialmente). Por otro lado, en Venezuela, sobresale la creciente radicalización del embate entre las fuerzas políticas centradas en la figura del gobierno y aquellas vinculadas a la oposición, que incrementa su pluralidad aunque de modo incipiente si comparado a Bolivia. En este cuadro, observamos la creciente incapacidad gubernamental para el diálogo y la paulatina articulación de los segmentos críticos internos.

En la primera sección, hacemos un breve análisis acerca de los términos hegemonía y populismo en el contexto de financiarización del capital. En seguida, exponemos separadamente las experiencias boliviana y venezolana, apuntando sus singularidades, la rearticulación de las fuerzas políticas y su reflejo en la composición del Congreso. En el caso de Bolivia, nuestra narrativa tiene como marco temporal la primera elección de Evo Morales, apuntando las fases del proceso de cambio, sus caminos y descaminos. En Venezuela, empezamos por la muerte de Hugo Chávez, cuando la disputa entre el oficialismo y la oposición recrudece, y hacemos un retorno a momentos que la precedieron. Ambos marcos representan un punto de tensión extrema entre las fuerzas políticas y evidencian los momentos distintos por lo que pasan las dos experiencias. Por fin, concluimos con una breve comparación entre los casos, apuntando sus diferencias y perspectivas futuras.

Hegemonía y populismo en la era de financiarización del capital: una hipótesis sobre la génesis de los gobiernos progresistas

Hasta el nuevo milenio, el populismo se expandió en el imaginário político latinoamericano como una categoría negativa. Sin embargo, este concepto ha sufrido una transformación radical, operada por Laclau (2005), asumiendo una perspectiva tanto descritiva cuanto normativa. Ambas características guardan relacción con los propósitos políticos de Laclau, en un contexto de lucha hegemónica impulsada en la región por una nueva élite política, la cual llega al poder en ese entonces. De esta manera, la categoría ha sido redefinida con el objetivo de enmarcar positivamente a estos nuevos actores, poniendo de relieve como elemento comun la recuperación del ideal nacional-desarrollista, recosntruído discursivamente a partir del rechazo a la agenda neoliberal y, sobre todo, de la polarización de la sociedad entre oprimidos y opresores (LACLAU, 2005).

Sin embargo, si en los populismo del pasado ya era posible detectar fronteras de antagonismo, en los nuevos populismos esas fronteras asumen una dimensión identitária, que se revela en la intención de reconocer actores que se mantuvieron en una posición de invisibilidad y subalternidad a lo largo de la historia. En esta nueva acepción, el líder populista no guía o lidera al pueblo, él lo representa por qué forma parte de él, una vez que comparte su identidad (ARDITI, 2005). 
Esos nuevos populismos tuvieron su génesis asociada a la indignación de las clases populares con las políticas neoliberales adoptadas en la región al final del siglo XX, pero su continuidad puede ser mejor comprendida por fatores sistêmicos, relativos a fluxos económicos internacionales. Esa nueva coyuntura, que eclode a principios de los años 2000 y perdura hasta 2014, está marcada por una elevación en los precios y en la demanda internacional por comodities, acelerada por el exponencial crecimiento chino. Su impacto político, por lo tanto, puede ser mejor comprendido a través del concepto de hegemonía, que se origina en la tradición marxista demarcando una inflexión en el debate teórico acerca de la relación entre estructura económica y superestructura política, enfatizando su carácter dialéctico. Con eso, nuestro objetivo es utilizar las conexiones entre política y economía como herramienta heurística para la comprensión de la génesis de los gobiernos de Evo Morales y Hugo Chávez, pero también para las dificultades encontradas en cuanto a su mantenimiento.

Al llevar a cabo este propósito, hay que considerar que, no obstante su dimensión estructural y prefiguradora del propio sistema internacional, la mayor vulnerabilidad y el carácter dependiente de las economías latinoamericanas no son atributos estáticos, sino vinculados a las oscilaciones inherentes a los ciclos de contracción y expansión de las economías globales (CAMPELLO, 2014). Esas oscilaciones (boom and bust economic cycles), por su vez, se agravan en el proceso de liberalización económica y financiera acelerada en las décadas finales del siglo XX (FRENCH-DAVIS, 1999).

Este proceso afecta en particular a las economías dependientes o periféricas, como las latinoamericanas, haciéndolas aún más expuestas a los ciclos de crecimiento y crisis, inherentes a la propia dinámica del sistema capitalista, pero más frecuentes en su fase financiera, debido a la mayor volatilidad de éste tipo de capital (BRESSER- PEREIRA, 2010). Las consecuencias de esta mayor exposición adquieren un nuevo nivel cuando se considera la estructura productiva de los países en cuestión, históricamente dependiente de la exportación de commodities. Por lo que una característica central de estos bienes es tener sus precios establecidos a través de dinámicas globales de mercado, aumentando la vulnerabilidad de sus productores a la coyuntura internacional.

Así, la consolidación de gobiernos progresistas en la región estaría asociada a un contexto internacional de elevación en los precios de las materias primas, mientras que su crisis estaría correlacionada con una coyuntura de retracción en los precios esos productos. Sin embargo, manteniendo un enfoque neogramsciano - que resalta las conexiones entre estructura y superestructura - hay que considerar las consecuencias políticas de estas transformaciones económicas. Estas, según el argumento aquí perseguido, están directamente relacionadas con el mayor o menor grado de autonomía relativa del sistema político frente a los intereses del mercado. Bajo esta perspectiva se observa que, cuando ocurre una elevación en los precios de los comodities (boom cycle), los fuertes ingresos de exportación disminuyen la demanda de los gobiernos por moneda extranjera para cumplir las obligaciones financieras externas. Al mismo 
tiempo que la aceleración del crecimiento económico mejora los índices de riesgo / retorno de las inversiones, haciendo que estas economías sean más atractivas para los inversores financieros.

Por lo tanto, considerando la mencionada correlación entre el mercado de commodities y los demás sectores del sistema financiero, en períodos de bonanza la rentabilidad de los inversores se encuentra relativamente asegurada en relación con eventuales sobrevuelos políticos. En este contexto, las élites financieras se ven menos interesadas a orientar sus esfuerzos (y recursos) para ejercer presiones sobre el sistema político, cuyos gobernantes pasan a gozar de mayor autonomía para perseguir proyectos menos convergentes como los intereses del mercado. Es decir, las elites políticas que gobiernan en estos momentos tienen un espacio más amplio para avanzar una agenda (populista) orientada al desarrollo ya la distribución del ingreso a las clases populares. Este fue el escenario que permitió la persecución de una serie de iniciativas de esa naturaleza a lo largo de la primera década del siglo XXI, no sólo en Venezuela y Bolivia, sino también en otros países de América del Sur.

La renovación del antagonismo político:

los desafíos al proceso de cambio en Bolivia.

En Bolivia, el agotamiento del modelo político-económico promocionado en la era neoliberal dió paso a la llegada al poder de un gobierno impulsionado por demandas populares y nacionalizantes en medio a la valorización de commodities en el mercado internacional y el aumento de exportaciones de recursos naturales desde Sudamérica. Así, las elecciones presidenciales de 2005 representaron una transformación, por lo menos inicialmente, en la configuración de fuerzas políticas y en la sociedad misma. Históricamente relegados al margen del bloque gobernante, al agro y a los servicios considerados menos prestigiosos en las urbes, los indígenas y campesinos finalmente parecían haber llegado al poder, convirtiéndose en protagonistas de un proceso norteado por el deseo de que se cambiara la lógica colonial. En que pesen casi dos siglos de independencia, los indígenas seguían como "extranjeros" dentro del territorio nacional, lo que pone en relieve las fallas de las democracias representativas liberales en reconocer las demandas de sus minorías.

Sea como sea, estos apuntes evocan la noción defendida por Laclau e Mouffle (1987) de que la democracia se caracteriza por su imposibilidad de inclusión total y, como tal, por una tensión inherente a su formación. Y, justamente por ser una "totalidad fracasada", esta tensión dará paso al surgimiento de demandas que, frente a la negligencia de sus gobernantes, pueden moverse con más fervor y abarcar tantas otras bajo una cadena de significación que una a los distintos sectores sociales que las plantean. En esta escena, figuran los "ingredientes" para el cambio en las relaciones de fuerza en que los de abajo destituyen a los antiguos gobernantes, aunque nada sea determinante ni que ocurra de manera automática. Es ahí donde se observan las disputas, las estrategias accionadas por los actores, las negociaciones, es decir, movimientos que constituyen el ethos de la política, según Laclau (2005). 
Estas palabras iniciales forman la plataforma para presentar al lector el momento paradigmático por el que ha pasado Bolivia. En este país, las fracturas y creciente marginación de la masa indígeno-campesina han llegado a principios del los años 2000 a un punto sin vuelta. Las medidas neoliberales, aplicadas desde mediados de los 80 paralelamente a la redemocratización, tuvieron entre sus consecuencias la profundización de la pobreza, el aumento del desempleo, de la inestabilidad política y de la inseguridad, conformando lo que Pizarro (2001) define como "vulnerabilidad social". Tales medidas, al mismo tiempo en que inserían Bolivia al panorama internacional del post Guerra Fría, expusieron el abismo en el tejido social boliviano, estructurado en la segregación económica y étnica entre sus integrantes. Además, prenunciaron una sensación de hartazgo en la gran parte de la población tras un periodo de intensa movilización social y represión por las fuerzas policiales. En el ámbito institucional, Bolivia contó con cinco presidentes entre 2001 y 2005 (ALEXANDRE, 2007, p.71).

La llegada de Evo Morales Ayma al Palacio Quemado, electo con $53.7 \%$ de los votos mientras el ex presidente Jorge "Tuto" Quiroga obtuvo $28,6 \%$, consistió, así, en un hecho emblemático, cuyo significado extrapola la sintomática crisis representación de la democracia en América Latina y la sustitución de un proyecto liberal la por otro más próximo al socialismo. En cuanto máximo líder del Movimiento al Socialismo-Instrumento Político por la Soberanía de los Pueblos (MAS-IPSP) - creado en los años 90 por cocaleros del Trópico de Cochabamba y por sindicatos y sectores de izquierda - Morales simbolizó la captura del poder por y a partir del indígena-campesino tras siglos de dominación. En 2006, mismo año de su posesión, el Presidente promulga as Leyes 3364 e 3365, que determinan, respectivamente, la convocatoria de la Asamblea Constituyente y la realización del referendo autonómico, dando paso a la "refundación del Estado". Este ente, a partir de la reforma constitucional y de intensos debates en la Asamblea, se convierte en Estado Plurinacional de Bolivia.

La Carta Constitucional condensa el intento de agregar un proyecto político que prevé, por un lado, el retorno del Estado como agente de transformación y promotor del desarrollo como condición para el ejercicio de la soberanía. Por otro lado, el documento propone la ampliación de la participación social por medio de mecanismos que garanticen a las organizaciones influir en las decisiones y diseños de políticas públicas. La noción de "plurinacionalidad" figura ahí como crucial, una vez que desplaza la idea de ciudadano como punto central del nuevo pacto social y la sustituye por las autonomías indígenas. Estas presuponen respeto a la autodeterminación y autogobierno de estas comunidades, ubicadas sea en ciudades o en territorios amenazados por la expansión del agroextrativismo $^{3}$ y los cultivos de coca. Este tenue equilibrio entre centralización y descentralización se convertirá en el foco de disputas, involucrando a los más distintos matices del juego político boliviano. Inicialmente, los embates ocurrirán entre el gobierno y su base aliada (indígeno-campesina), de un lado, y la oposición, compuesta por los políticos provenientes de los antiguos partidos (empresarios, terratenientes), en el otro extremo. Posteriormente, las disputas serán cruzadas por desentendimientos en el
3. Según Acosta (2011), el extractivismo comprende actividades de extracción de recursos naturales (aunque limitadamente procesados) en grandes volúmenes para la exportación, como la minería, el sector de hidrocarburos, monocultivos, la explotación de madera. 
4. El Oriente Boliviano es compuesto por los departamentos de Santa Cruz de la Sierra, Beni, Pando, donde se concentran las actividades extractivistas. Junto a Tarija y Chuquisaca, estos 5 departamentos forman la llamada Media Luna cuyos políticos y cívicos plantearon sus proyectos autonómicos ceno de los aliados, estableciendo un escenario que divide a los líderes y organizaciones indígenas, como analizaremos adelante.

Las disputas frente al enemigo común

El primer gobierno Morales (2006-2009) fue caracterizado por una serie de cambios institucionales. En este periodo de la llamada "Revolución Cultural", la administración adopta iniciativas como la nacionalización de los hidrocarburos. Otra medida importante fue la promoción del Programa de Reforma Agraria, a través de la cual el Ejecutivo promocionaba la distribución de tierras y territorios indígenas paralelamente a la manutención del latifundio, aunque lo condicionaba a la comprobación de su "Función Económica Social" (FES). Estas políticas se inserían en un cuadro que proponía romper una situación de dependencia económica de Bolivia y transcender la lógica colonial representada por la vieja élite dominante.

A esta retórica descolonizadora y revolucionaria, reproducida por el gobierno y muchos de los movimientos sociales que le apoyaban, se sumaban la anti-imperialista y anti-americanista. En esta cadena de significación, Estados Unidos y las tradicionales élites bolivianas racistas, que se contraponían a las transformaciones buscadas por la administración Morales, eran identificados como los "enemigos del proceso de cambio". También, eran caracterizados como "colonizadores", "criollos-mestizos", "usurpadores" de las tierras y territorios indígenas (DELGADO, 2014). Tal identificación evidenciaba, de esta manera, el antagonismo que perfila la política y las disputas a la construcción de la hegemonía, conforme teorizó Laclau.

De hecho, los embates entre estos dos campos del espectro político no tardaron a mostrarse de manera abierta y violenta, especialmente en el departamento de Santa Cruz de la Sierra, bastión de la oposición del Oriente Boliviano ${ }^{4}$. Así, entre 2005 y 2007, se observó una serie de agresiones practicadas por miembros de la élite cruceña contra campesinos e indígenas. También en 2007, la élite de Cochabamba, municionada de palos, bastones, acosa y agrede a los últimos. Al año siguiente, algo similar ocurre en Chuquisaca, cuando políticos y jóvenes estudiantes contrarios al MAS amenazan quemar vivos a campesinos que se encontraban en la capital Sucre para recibir ambulancias del gobierno. Así como en Cochabamba y Santa Cruz, en Chuquisaca los indígenas y campesinos eran identificados por las élites como "masistas" o afines al partido gubernamental, como señala Delgado (2014). A estos eventos sucedió la desarticulación de lo que la Inteligencia Boliviana anunció como un plan para asesinar a Morales y a Álvaro García, en el episodio conocido como "asalto al Hotel las Americas". El plan habría sido auspiciado por las élites del agro con el apoyo norte-americano y fue noticiado como un intento de golpe.

Todos estos acontecimientos ocurrieron y fueron motivados por el tema de las autonomías departamentales, demandada por las élites del Oriente como una contraposición a las autonomías indígenas en cuanto representativa de un proyecto político que les alejaba de la arena boliviana. Aunque la idea se mezcle a otras demandas localizadas, el tema de las autonomías departamentales ganó fuerza frente al empoderamiento de 
los de abajo y sus intentos de trastornar el orden institucional anterior. Como forma de asegurarse en el poder y resistir a este cambio en las relaciones de fuerza, las antiguas élites buscaron defender una propuesta que les garantizara el manejo político y económico en los departamentos. Los embates entre gobierno y oposición en la primera gestión de Morales reflejan, en este sentido, las disputas a la construcción de la hegemonía bajo la llave centralización-descentralización y que, en realidad, se fundan en relaciones estructurales de sociedad boliviana.

El quiebre en el seno del bloque popular

El segundo gobierno Morales y primero del Estado Plurinacional (2010-2014) significó un retroceso en la medida en que se revertieron importantes logros alcanzados por el bloque popular. Es en este momento que los desacuerdos ya presentes entre las distintas organizaciones de la base aliada emergen una vez que la figura del "enemigo común", que unía a actores con demandas sectoriales por veces contradictorias, pierde fuerza. De hecho, tras las crecientes derrotas políticas, de las cuales la fundación del Estado Plurinacional resulta como el más contundente, el asalto al Hotel Las Américas representó el "golpe maestro" del gobierno: al promover el debilitamiento de la oposición y su aislamiento en la escena política, la administración Morales se fortalece y va ganando espacio en el Oriente, especialmente en Santa Cruz. En este proceso, el acercamiento al gobierno - que promociona incentivos a la economía del agro - se presentará de momento como la única alternativa a la sobrevivencia de la oposición, aunque que esto no signifique una alianza. La oposición del agroextractivismo se mantendrá pendiente para hacer frente, siempre que posible, al oficialismo y lo hará por ocasión de la tensión entre el Ejecutivo y los indígenas comunarios", cuando el "gobierno de los movimientos sociales" revela su autoritarismo en defensa de los proyectos desarrollistas.

Luis Tapia (2011) y Raúl Prada (2014), asocian este cambio de imagen al "gasolinazo", una serie de protestas ocurridas en 2010 alrededor de Bolivia contra la medida gubernamental de cancelar el subsidio estatal al los carburantes, llevando a un aumento generalizado de precios. Sin embargo, la postura intransigente de la administración se mostrará de manera incontestable el año siguiente, en la represión a los comunarios que participaban de la VIII Marcha Indígena. La marcha había sido organizada por líderes indígenas para interrumpir la construcción de una carretera que cruzaría el Territorio Indígena Parque Nacional Isiboro-Sécure (TIPNIS). El proyecto, ejecutado por el gobierno sin el establecimiento de una consulta previa a las comunidades indígenas, viola el derecho constitucional a la autonomía indígena, también asegurado por las normas internacionales, como el Convenio 169 de la Organización Internacional del Trabajo (OIT). Por eso, este episodio y los embates que se siguieron por la construcción de la vía sobresalen como una contradicción del proceso de cambio a la vez que revierten el empoderamiento indígena y subordina este actor político otra vez a los mandos del Estado (TAPIA, 2011; PRADA, 2014).
5. Los indígenas comunarios son aquellos que viven en comunidades, en territorios indígenas. Estos actores están agrupados en 2 grandes organizaciones: el Consejo Nacional de Ayllus y Markas del Qullasuyu (Conamaq) y la Confederación de los Pueblos Indígenas del Oriente, Chaco y Amazonía de Bolivia (Cidob), representantes de altiplano y las tierras bajas bolivianas, respectivamente. 
Otro factor relevante consiste en las divisiones en el seno de los movimientos indígenas a lo largo del país, desplazando el antagonismo antes asistido entre la base aliada y la oposición de la Media Luna hacia el centro del bloque popular. En este momento, se desplaza también la figura del enemigo: mientras la administración Morales y sus aliados promueven una equivalencia entre la antigua oposición y los indígenas contrarios a la carretera, los últimos verán al gobierno como el enemigo (DELGADO, 2017).

Lucha hegemónica y élites parlamentarias:

las etapas del proceso de cambio en la Asamblea de Bolivia

A los momentos evaluados previamente corresponden cambios en la composición de las fuerzas parlamentarias, lo que demostrará la paulatina ampliación de la base gubernista en la Asamblea Legislativa. En ese sentido, en las elecciones de 2005, de los 27 escaños en el Senado, el MAS obtiene 12 , mientras los demás estarán en manos de la tradicional élite dominante, representada por Poder Democrático Nacional (Podemos), Movimiento Nacional Revolucionario (MNR) y Unidad Nacional (UN). Aunque la mayoría lograda por estos partidos políticos en el Senado reflejaba la permanencia de antiguos actores en la arena política, la creación de Podemos y UN también apuntaba la necesidad de reinvención de esta misma élite, adecuándose al los "nuevos tiempos". Por eso, políticos de corte neoliberal y pertenecientes a la oligarquía boliviana, como "Tuto" Quiroga y el empresario Samuel Doria Medina, impulsaron la fundación de estos dos partidos, respectivamente. El escenario político en esta casa parlamentaria resultará en una fuerte tensión con el Ejecutivo que, frente a la oposición de los senadores, buscará implementar sus proyectos vía la promulgación de Decretos Supremos. Ya en la Cámara de Diputados, el MAS logra ocupar 72 de los 130 escaños, divididos entre diputados uninominales (electos por sufragio directo) y plurinominales (electos en proporción a los votos obtenidos por cada partido político), como se señala en la tabla abajo:

Tabla 1: Elecciones Parlamentarias de 2005 en Bolivia

\begin{tabular}{c|c|c|c}
\hline \multicolumn{5}{c}{ Cámara de Diputados } \\
\hline Uninominales & Plurinominales \\
\hline Partido & Escaños & Partido & Escaños \\
\hline MAS & 45 & MAS & 27 \\
\hline Podemos & 22 & Podemos & 21 \\
\hline MNR & 2 & MNR & 5 \\
\hline UN & 1 & UN & 7 \\
\hline Total & 70 & Total & 60 \\
\hline Senado & & & Escaños \\
\hline Partido & & & 13 \\
\hline Podemos & & & 1 \\
\hline MAS & & & 27 \\
\hline MNR & & & \\
\hline UN & & & \\
\hline Total & & & \\
\hline
\end{tabular}

Fuente: elaboración propia usando datos del Political Database of the Americas, Duke University 
Las normativas del Estado Plurinacional de Bolivia, a través del Régimen Electoral Transitorio (Ley 4.021), introdujeron modificaciones diversas en el sistema electoral, entre ellas: el número de escaños para el Senado es ampliado de 3 para 4 senadores por departamento. En la Cámara de Diputados, a los 70 escaños uninominales se suman 7 , destinados a los representantes de los pueblos indígenas (1 escaño por departamento), electos bajo sus usos y costumbres. Para algunos analistas, este número reducido de escaños, que también ha sido motivo de tensiones dentro de la base aliada, pone en duda la propia "plurinacionalidad" ya que, en Bolivia, se reconocen oficialmente a 36 naciones (ALMARAZ, A. et al, 2011). En las elecciones de 2009, bajo la Nueva Constitución, de los 36 escaños al Senado el MAS obtiene 26, mientras elige a 88 de los 130 diputados que conforman la Cámara Baja. El control del Legislativo asegura al gobierno mayor margen de maniobra, aunque su actuación sea sometida a tensiones en el ceno de la base aliada, como ya señalamos.

Tabla 2: Elecciones Parlamentarias de 2009 en Bolivia

\begin{tabular}{|c|c|c|c|}
\hline \multicolumn{4}{|c|}{ Cámara de Diputados } \\
\hline \multicolumn{2}{|c|}{ Uninominales } & \multicolumn{2}{|c|}{ Plurinominales } \\
\hline Partido & Escaños & Partido & Escaños \\
\hline MAS & 49 & MAS & 33 \\
\hline PPB-APB & 19 & PPB-APB & 17 \\
\hline AS & 2 & $\mathrm{UN}-\mathrm{CP}$ & 3 \\
\hline Total & 70 & Total & 53 \\
\hline \multicolumn{4}{|c|}{ Uninominales por Circunscripciones Especiales (indígenas) } \\
\hline \multicolumn{2}{|c|}{ Partidos } & \multicolumn{2}{|c|}{ Escaños } \\
\hline MAS & & & 6 \\
\hline PPB-APB & & & 1 \\
\hline Total & & & 7 \\
\hline \multicolumn{4}{|c|}{ Senado } \\
\hline \multicolumn{2}{|c|}{ Partidos } & \multicolumn{2}{|c|}{ Escaños } \\
\hline MAS & & & 26 \\
\hline PPB-APB & & & 10 \\
\hline Total & & & 36 \\
\hline
\end{tabular}

Fuente: elaboración propia usando datos del Political Database of the Americas, Georgetown University

Los resultados de las elecciones de 2014 parecen confirmar, por lo menos en términos cuantitativos, el predominio de la nueva élite política y de su proyecto en la escena boliviana en medio a cambios en la distribución de escaños en la Cámara y a la unión de distintas fuerzas de la oposición (AZCUI, 2014). Así surge Unidad Demócrata (UD), a partir de la fusión entre UN y el Movimiento Democracia Social (Democratas), partido de centroderecha creado por la tradicional élite de la Media Luna. En ese momento, el MAS elige a 88 de los 130 diputados y a 25 de los 36 senadores, manteniéndose como la gran fuerza política en el país. Sin embargo, la "victoria" del partido gubernista será puesta en jaque por los resultados de las elecciones subnacionales de 2015. 
Tabla 3: Elecciones Parlamentarias de 2014 en Bolivia

\begin{tabular}{|c|c|c|c|}
\hline \multicolumn{4}{|c|}{ Cámara de Diputados } \\
\hline \multicolumn{2}{|c|}{ Uninominales } & \multicolumn{2}{|c|}{ Plurinominales } \\
\hline Partido & Escaños & Partido & Escaños \\
\hline MAS & 49 & MAS & 33 \\
\hline UD & 12 & UD & 19 \\
\hline PDC & 2 & PDC & 8 \\
\hline Total & 63 & Total & 60 \\
\hline \multicolumn{4}{|c|}{ Uninominales por Circunscripciones Especiales (indígenas) } \\
\hline \multicolumn{2}{|c|}{ Partidos } & \multicolumn{2}{|c|}{ Escaños } \\
\hline MAS & & & 6 \\
\hline UD & & & 1 \\
\hline Total & & & 7 \\
\hline \multicolumn{4}{|c|}{ Senado } \\
\hline \multicolumn{2}{|c|}{ Partidos } & \multicolumn{2}{|c|}{ Escaños } \\
\hline MAS & & & 25 \\
\hline UD & & & 9 \\
\hline PDC & & & 2 \\
\hline Total & & & 36 \\
\hline
\end{tabular}

Fuente: elaboración propia usando datos del Tribunal Supremo Electoral (TSE) de Bolivia

¿La consolidación del gobierno?

El tercer gobierno Morales, que empieza en 2015, podría reflejar la consolidación del oficialismo en Bolivia, de su hegemonía sí se toman en cuenta los porcentajes de voto a favor del Presidente y su vice, $61,04 \%$, y la mayoría en el Congreso. En ese sentido, se podría suponer la formación de un consenso en torno al proyecto de Estado encabezado por Morales y, por lo tanto, el debilitamiento de la oposición, ahora formada tanto por la antigua élite dominante como por los disidentes del bloque popular y del MAS. Sin embargo, la presunta "victoria" del gobierno es puesta en jaque delante de los resultados de las elecciones municipales y departamentales. En que pese la expresiva votación en el interior del país, el MAS no logra elegirse en las principales capitales de Bolivia. En Santa Cruz de la Sierra, vencen los políticos de la oligarquía tanto para la gobernación cuanto para la capital, reforzando la posición contraria del departamento al gobierno. Algo semejante ocurre en Tarija, con la victoria de la oposición en el departamento y la capital, aunque en Pando, Beni, Chuquisaca, Cochabamba y Potosí el MAS haya elegido sus gobernadores.

En El Alto, uno de los bastiones del gobierno, vence la descendiente de aymaras Soledad Chapetón por UN. Para la gobernación de La Paz, antes presidida por el masista César Cocarico, vence el representante de la agrupación ciudadana Soberanía y Libertad Bolivia (SOL.bo), el aymara Félix Patzi, quien dejó al MAS en 2010. Desde entonces, el ex-ministro de Educación y proponente del llamado "tercer sistema" (o "sistema comunal") se ha establecido como una das las más importantes alternativas políticas a Evo Morales, publicando sus pensamientos en libros y periódicos bolivianos (PATZI, 2013 y 2013a). Después del Presidente, Patzi es el 
primer político cuya posesión fue organizada por comunarios del Altiplano, siguiendo las ceremonias tradicionales aymaras, lo que simboliza la relevancia que los mismos le atribuyen. También la ciudad de La Paz siguió el voto contrario al oficialismo, así como la capital de Cochabamba. Con relación a las demás capitales, los gubernistas se mantuvieron apenas en Sucre y Potosí.

Por un lado, estos resultados revelan el crecimiento del partido gubernista que, a partir de su ascensión al Palacio Quemado, logra establecerse en las dos cámaras del Legislativo y en las esferas municipales y departamentales, abarcando la gran parte del país. Por otro lado, la expansión del MAS, impulsada por el Ejecutivo y sus bases, demuestra sus límites en la pérdida de poder en centros importantes. Este punto releva igualmente la dificultad del partido en sostener una estrategia que busca sobreponer la figura de candidatos masistas a la de Evo Morales, muchos de los cuales todavía no se firmaran como políticos hábiles como el presidente. De hecho, la falta de cuadros que lleven adelante el proyecto oficialista ya había sido señalada por líderes del MAS en otros momentos, por lo que se creó la Escuela de Formación Política del partido, en mayo de 2012 (CLAROS, 2012). Este es el periodo en que los desacuerdos dentro del bloque popular resultaron en el alejamiento de antiguos aliados y su vinculación a partidos políticos opositores ya existentes o recién creados.

Respecto a las tensiones mencionadas anteriormente, los comunarios de las tierras bajas que se contraponen a la carretera siguen presionando: luego de haber denunciado el caso a la OEA, los indígenas decidieron alistar una marcha desde Estados Unidos a Bolivia frente a las afirmaciones del Presidente sobre la construcción de la vía (ARIÑEZ, 2015). Todos estos hechos apuntan no sólo para la permanencia de una popularidad centrada en la figura de Evo Morales, y no en el partido oficialista, como señala Molina (2015), sino también demuestran que el gobierno deberá seguir negociando en frentes distintos para mantenerse en el poder. Este tema parece confirmarse al año siguiente, cuando los bolivianos rechazan por $51,30 \%$ contra $48,70 \%$ la reforma constitucional propuesta por el gobierno, que permitiría la segunda reelección de Morales bajo las normas del Estado Plurinacional (TSE, 2016). En ese sentido, aunque el "No" haya ganado por un margen estrecho, los resultados reiteran la debilidad de la estrategia gubernamental, que busca sostener proyecto hegemónico del MAS anclándolo a la popularidad del mandatario, por sí misma incapaz de asegurarlo. Así, contrariamente a la idea de consolidación del oficialismo, el fortalecimiento de la oposición sugiere la necesidad del gobierno de hacer más esfuerzos para alcanzar el equilibrio en el juego político boliviano.

La división del chavismo y la purga de Maduro

En 05 de marzo de 2013, el líder revolucionario de Venezuela, Hugo Rafael Chávez Frías, murió en Caracas. El entonces vicepresidente Nicolás Maduro, uno de sus colaboradores más cercanos de los últimos 14 años de revolución bolivariana, fue el encargado de anunciar su muerte y asumir la difícil tarea de continuar su legado. El 14 de abril, ocurrie- 
6. Esta no es la primera vez que alguna organización con fines políticos es rechazada en su intento por inscribirse en el CNE. En 2010, el partido Voluntad Popular, de Leopoldo López, experimentó el mismo caso, cuando le fue negada la inscripción. ron los comicios para elegir al nuevo presidente de Venezuela. Maduro, compitió contra el líder de la oposición, Henrique Capriles Radonsky. Su performance, sin embargo, no fue tan buena como la de su antecesor, que había obtenido el 55\% de los votos, en las elecciones presidenciales de Venezuela de octubre de 2012, cuando Hugo Chávez fue reelecto para un tercer mandato consecutivo, mientras que el mismo Capriles alcanzó $44,30 \%$. La oposición vociferó que le habían robado la elección y se fue a las calles para impugnar la elección, a las que también se volcaron los chavistas para defender a Maduro. Hubo disturbios por varios días y el saldo final fue de 7 muertos y 60 heridos.

Poco más de un año después, en el III Congreso del Partido Socialista Unido de Venezuela (PSUV), Diosdado Cabello, primer vicepresidente del PSUV y principal oponente de Maduro en disputa interna por la hegemonía del bloque chavista, al anunciar los delegados electos añadió que el objetivo del encuentro es transformar la organización "no solo en un partido que sea capaz de ganar elecciones sino que sea capaz de hacer una revolución y además una revolución socialista, una revolución bolivariana"(EL NACIONAL, 2014). Sin embargo, a pesar del esfuerzo de presentarse como un bloque monolítico - incluso superando incluso la saludable separación el gobierno y partido - es demasiado significativo que, en la reunión preparatoria para el Congreso, el presidente del partido Nicolás Maduro tenga dedicado más tiempo a los "traidores" que a los desafíos de la revolución.

El mensaje tiene una dirección clara si tenemos en cuenta que, en el mes anterior, el chavismo había sufrido dos grandes pérdidas, con la destitución del ministro de Planificación y personaje histórico del chavismo, Jorge Giordani que respondió a su exoneración con una carta abierta criticando a Maduro por su incompetencia en el manejo de la economía y sus debilidades de liderazgo. Profundizando la división en el bloque chavista, a ello le siguió una carta crítica de apoyo al ex ministro por parte de otra estrella del chavismo, Héctor Navarro, a posteriori suspendido de su cargo directivo en el PSUV. Giordani y Navarro actuaron directamente junto a Chávez en la redacción de la Agenda Alternativa Bolivariana, publicada en julio de 1996, documento histórico, esencial para edificar las bases económicas, políticas y ideológicas del bolivarianismo chavista. La persecución de los dos personajes es una fuerte señal de la disminución de la influencia del ala marxista radical en temas de política económica y de la falta de cohesión interna en el oficialismo.

En noviembre del mismo año, ocurre una nueva cisión, ahora en el interior de PSUV. Un grupo de miembros de la juventud del partido solicitó registrarse junto al Consejo Nacional Electoral (CNE) bajo el nombre de Marea Socialista. El CNE negó la inscripción de Marea y otras nueve organizaciones que tendrían que buscar un nombre legal diferente para intentar inscribirse ${ }^{6}$, hasta el día previo al inicio del período de candidaturas para las elecciones a la Asamblea Nacional, convocadas para el 6 de diciembre de 2015 (EL UNIVERSAL, 2015). Marea Socialista se declara "chavista, socialista" y niega que su propósito sea dividir el chavismo, aunque sí erosiona el apoyo a la cúpula del PSUV vinculada con la corrupción. Según Nicmer Evans, uno de los fundado- 
res de la agremiación, hay una crisis de hegemonía también dentro de dos bloques, lo que genera la creación de nuevos movimientos críticos de la forma en que el liderazgo se ha ejercido en la oposición y en el chavismo, cuyo propósito es representar a los que ya no se identifican con las cúpulas.

Estas "traiciones" - y la acción cada vez más implacable del Tribunal Disciplinario ${ }^{7}$ - evidencian que el PSUV está perdiendo su lugar de partido hegemónico, un lugar dado no solo por ganar elecciones sino fundamentalmente por ser capaz de generar consenso interno. La declaración de Evans pone de relieve la erosión en los dos polos de la lucha hegemónica en Venezuela, teniendo en cuenta una disputa por la hegemonía dentro de cada bloque y no sólo entre ellos. Además llama atención a un grupo cada vez mayor de ciudadanos que no se identifican a sí mismos ni con el chavismo ni la con oposición (los llamados ni-ni). El problema para Maduro es que para emprender una purga a los traidores del partido es necesario tener una gran concentración de poder político, o una gran dosis de éxito económico. En la situación venezolana actual ambos aparecen poco probables. Su presidencia tiene un déficit congénito de autoridad y la economía no muestra signos de recuperación. Continúan la persistente inflación con estancamiento, la total ausencia de inversión privada y la carencia de bienes de primera necesidad.

Salidistas vs dialogadores: el atasco en la oposición venezolana

El año de 2014 empezó en Venezuela con una operación emprendida por un sector oposicionista denominado "La Salida" que, tras varios meses de manifestaciones y protestas, se concluyó con 43 personas muertas, cuarenta opositores en prisión y más de 600 heridos. Dicha operación comenzó en enero del mismo año, impulsada por la ex diputada María Corina Machado y el dirigente del partido Voluntad Popular, Leopoldo López. Su objetivo, según los organizadores, seria invitar a los venezolanos a protestas pacíficas para debatir una forma para poner fin al gobierno del presidente, Nicolás Maduro, sin tener que esperar hasta las próximas elecciones (GOULART, 2014).

La opción por una estrategia insurreccional ha precipitado la división del bloque oposicionista, sobretodo con el ala moderada que controla la Mesa de la Unidad Democrática, alianza de organizaciones políticas opuestas al gobierno, bajo el liderazgo su principal estrella: Henrique Capriles Radonsky.

Desde las elecciones presidenciales y municipales de 2013, cuando fue doblemente derrotado por el chavismo, el gobernador de Miranda ha insistido en que salir a las calles no es la mejor forma de abordar la causa opositora. Desde entonces, ha liderado una agenda de diálogo con el gobierno para fijar objetivos comunes. Sin embargo, aún hoy día se proclame un defensor de la Constitución de 1999, Capriles fue un activo integrante del asedio a la embajada de Cuba ocurrido en el 12 de abril de 2002, día siguiente del derrocamiento de Hugo Chávez por militares y civiles adversos a su persona. Después de haber investido en la Presidencia al líder de Fedecámaras Pedro Carmona Estanga, se
7. Según se informa en su website, donde es ampliamente estimulado la denuncia de "traidores", el Tribunal Disciplinario "es la instancia encargada de sancionar a aquellos militantes que incumplan con sus deberes formales dentro de la tolda o realicen acciones que acarreen algún perjuicio contra el PSUV" (Ver: http://www.psuv.org.ve/ tribunal-disciplinario/) 
produjeron actos de intimidación por parte de los golpistas, contra el personal de la embajada cubana, entre otros disturbios que ocasionaron más de 20 muertos y 100 heridos, en los cuales también se enredó Leopoldo López. En 2012, López fue el precandidato presidencial en las primarias de la oposición, pero se retiró al comprobar sus escasas oportunidades de derrotar al favorito de la contienda Henrique Capriles. En 2014, quedaría nuevamente habilitado para correr por cualquier cargo público, pero decidió adelantarse y solicitar en la calle un cambio inmediato de gobierno.

En el marco de esta campaña, el ala radical de la oposición rompe con los moderados y convoca una marcha el 12 de febrero. Además de inúmeros heridos, tres personas fueron muertas: dos opositores y un chavista. En los barrios caraqueños de clase media y más diez ciudades del país, gobernadas por la oposición, las manifestaciones se replicaron durante cuatro meses. Por "sus llamados a la sedición" y teniendo en cuenta su supuesta responsabilidad en la violencia, fue librada una orden de captura contra López, que se entregó a las autoridades, seis días después del inicio de las protestas.

En un intento de poner fin a la escalada de violencia, en 08 de abril de 2014, cercados por ocho cancilleres de América del Sur, el gobierno de Nicolás Maduro y la alianza opositora acordaron iniciar un proceso de diálogo. Cabe subrayar que varios partidos de la oposición - entre ellos Voluntad Popular, Comité de Política Electoral Independiente (Copei), Causa R y Proyecto Venezuela- se abstuvieron de participar aduciendo diferentes razones. Estas renuencias son un aviso que la falta de cohesión que amenaza la supervivencia del chavismo también es un problema para sus enemigos.

Lucha hegemónica y élites parlamentarias: las etapas del proceso de cambio en la Asamblea Nacional Venezolana

Con respecto a la lucha hegemónica librada en Venezuela, es posible destacar el papel de los procesos electorales, que, en consonancia con el núcleo normativo de la Carta de 1999, se caracteriza por el énfasis en el poder constituyente. Por lo tanto, el impresionante éxito electoral del chavismo, victorioso en todas las disputas, con la excepción del referéndum 2007, puede ser apuntado como el principal instrumento del oficialismo en la lucha hegemónica trabada en el país.

De acuerdo con el argumento de que aquí se presenta, tales victorias, abajo agregadas en una tabla, ofrecen al chavismo un manto de legalidad y un soporte institucional que disminuye su disposición a negociar y dialogar con las minorías parlamentarias, reduciendo la Asamblea al papel de una instancia de aclamación las decisiones tomadas por el Ejecutivo. Además, su buen desempeño electoral afecta las estrategias de la oposición, que se alterna entre la movilización electoral, cuando alimenta esperanzas de derrotar al gobierno a través de elecciones (executivas, legislativas y de referendos revocatorios), y la insurrección golpista, cuando se percibe derrotada por el éxito del chavismo en las urnas. 
Tabla 4: Procesos electorales celebrados bajo la Constitución de 1999

\begin{tabular}{|c|c|c|}
\hline Año & Tema & Resultado \\
\hline 1998 & Elecciones presidenciales & $\begin{array}{l}\text { Chávez ganó con un } 56 \% \text { de los votos frente al } 40 \\
\% \text { de Henrique Salas Romer. }\end{array}$ \\
\hline 1999 & $\begin{array}{l}\text { Referendo constituyente } \\
\text { de abril }\end{array}$ & $\begin{array}{l}\text { Alrededor de un } 90 \% \text { de los votantes decidió con- } \\
\text { vocar a una Asamblea Constituyente para redactar } \\
\text { una nueva carta magna. La abstención llegó al } 62 \\
\% \text {. }\end{array}$ \\
\hline 1999 & $\begin{array}{l}\text { Referendo constituyente } \\
\text { de diciembre }\end{array}$ & $\begin{array}{l}\text { El } 72 \% \text { de los votantes aprobó la nueva Consti- } \\
\text { tución, lo que dio inicio a la llamada "Revolución } \\
\text { Bolivariana". La nueva Constitución determinó la } \\
\text { unicameralidad del Congreso -hoy Asamblea Na- } \\
\text { cional. }\end{array}$ \\
\hline 2000 & $\begin{array}{l}\text { Elecciones presidencia- } \\
\text { les, regionales, municipa- } \\
\text { les y parlamentarias }\end{array}$ & $\begin{array}{l}\text { Chávez obtuvo el } 59,76 \% \text { de los votos frente al } \\
37,5 \% \text { de su principal contendor, Francisco Arias, } \\
\text { para el mandato que inició en el } 2001 .\end{array}$ \\
\hline 2004 & Elecciones regionales & $\begin{array}{l}\text { El oficialismo ganó } 22 \text { de los } 24 \text { estados y más del } \\
80 \% \text { de las alcaldías. La oposición se debilitó, al } \\
\text { perder seis de las ocho gobernaciones que con- } \\
\text { trolaba y la gran mayoría de las } 220 \text { alcaldías que } \\
\text { mantenía desde el } 2000 \text {. }\end{array}$ \\
\hline 2004 & $\begin{array}{l}\text { Referendo revocatorio } \\
\text { sobre la permanencia del } \\
\text { presidente }\end{array}$ & $\begin{array}{l}\text { En agosto, el } 59 \text { \% de los casi } 10 \text { millones de votan- } \\
\text { tes decidieron que Chávez debía seguir en el poder } \\
\text { en un referéndum activado por la oposición para } \\
\text { revocar su mandato. }\end{array}$ \\
\hline 2005 & $\begin{array}{l}\text { Elecciones parlamenta- } \\
\text { rias }\end{array}$ & $\begin{array}{l}\text { Los } 165 \text { escaños de la Asamblea fueron ganados } \\
\text { por el gobernante Movimiento Quinta República } \\
\text { (MVR) o partidos simpatizantes de Chávez, luego } \\
\text { de que la oposición se retirara de la contienda ale- } \\
\text { gando falta de confianza en el árbitro electoral. La } \\
\text { abstención fue del } 75 \% \text {. }\end{array}$ \\
\hline 2006 & Elecciones presidenciales & $\begin{array}{l}\text { Chávez fue reelegido con un } 62 \% \text { de los sufragios } \\
\text { frente al } 37 \% \text { del candidato opositor Manuel Ro- } \\
\text { sales, prometiendo llevar a Venezuela hacia el "So- } \\
\text { cialismo del Siglo XXI". }\end{array}$ \\
\hline 2007 & Referendo constitucional & $\begin{array}{l}\text { Chávez sufrió su primera derrota electoral cuando } \\
\text { su propuesta para reformar la Constitución y pro- } \\
\text { fundizar su proyecto socialista fue rechazada por } \\
\text { un muy estrecho margen. }\end{array}$ \\
\hline 2008 & Comicios regionales & $\begin{array}{l}\text { Se realizaron a fines de noviembre para elegir } 603 \\
\text { cargos entre gobernadores, alcaldes y concejales. } \\
\text { Aunque la oposición ganó emblemáticos estados y } \\
\text { alcaldías, el oficialista PSUV logró conquistar nue- } \\
\text { vamente la mayoría de los cargos en juego. }\end{array}$ \\
\hline 2009 & $\begin{array}{l}\text { Referendo sobre enmien- } \\
\text { da constitucional }\end{array}$ & $\begin{array}{l}\text { A fines del 2008, Chávez propuso un referéndum } \\
\text { para eliminar los límites a la reelección de cargos } \\
\text { públicos fijados en la Constitución de } 1999 \text {. La vo- } \\
\text { tación se llevó a cabo en febrero del } 2009 \text { y el "Si" } \\
\text { ganó con casi el } 55 \% \text { de los votos. }\end{array}$ \\
\hline 2010 & Elecciones legislativas & $\begin{array}{l}\text { En septiembre fueron elegidos los miembros de la } \\
\text { Asamblea Nacional (AN) hasta el 2016. El oficia- } \\
\text { lismo ganó la mayoría de escaños pero no alcan- } \\
\text { zó los dos tercios de los escaños requeridos para } \\
\text { aprobar grandes reformas. }\end{array}$ \\
\hline
\end{tabular}




\begin{tabular}{l|l|l}
\hline 2012 & Elecciones presidenciales & $\begin{array}{l}\text { El } 7 \text { de octubre Chávez fue reelecto con el 55,07\% } \\
\text { de los votos. El opositor Henrique Capriles logró } \\
\text { el 44,31\%. A pesar de que el mandatario obtuvo } \\
\text { una cómoda victoria, la oposición logró su mejor } \\
\text { resultado en más de una década al alcanzar 6,5 } \\
\text { millones de votos. }\end{array}$ \\
\hline 2012 & Elecciones regionales & $\begin{array}{l}\text { En diciembre el PSUV obtuvo 20 de las } 23 \text { gober- } \\
\text { naciones en juego, a pesar de la ausencia del presi- } \\
\text { dente Chávez quien para ese momento convalecía } \\
\text { en Cuba. }\end{array}$ \\
\hline 2013 & Elecciones municipales & $\begin{array}{l}\text { Primer proceso electoral posterior a la muerte de } \\
\text { Hugo Chávez. Nicolás Maduro ganó estos comi- } \\
\text { cios por el 50.61 \% de los votos, mientras, el oposi- } \\
\text { tor, Henrique Capriles obtuvo 49,12\%. }\end{array}$ \\
\hline $\begin{array}{l}\text { Los resultados indican una victoria del PSUV y } \\
\text { sus aliados en la mayoría de los municipios del } \\
\text { país, ya que obtuvieron 240 de las 337 alcaldías, } \\
\text { pese que las grandes ciudades quedaron regidas } \\
\text { por candidatos opositores. El chavismo también } \\
\text { obtuvo la mayoría de los concejos municipales y } \\
\text { la mayoría de votos a nivel nacional (48,69\% para } \\
\text { la alianza oficialista lo que representa mientras } \\
\text { que la alianza opositora MUD obtuvo 39,34\% de } \\
\text { los votos). }\end{array}$ \\
\hline
\end{tabular}

Fuente: El Impulso.

Por otra parte, en lo que respecta a la legislatura, el éxito electoral ha permitido al chavismo el transito libre en la Asamblea, desde las elecciones de julio de 2000, cuando se sufragó en simultáneo los miembros del Poder Legislativo y Executivo. En las parlamentarias, el entonces Movimiento Quinta Republica (MVR) que más tarde dará paso al Partido Socialista Unido de Venezuela (PSUV), también obtuvo una significativa victoria. Como se señala en la tabla de abajo:

Tabla 5: Elecciones Parlamentarias de 2000 en Venezuela

\begin{tabular}{c|c}
\hline Partido & Escaños \\
\hline MVR & 92 \\
\hline AD & 33 \\
\hline PV & 6 \\
\hline COPEI & 6 \\
\hline MAS & 6 \\
\hline LCR & 3 \\
\hline PJ & 5 \\
\hline CONVERGENCIA & 4 \\
\hline UNT & 3 \\
\hline PPT & 1 \\
\hline Otros & 7 \\
\hline Total & 165 \\
\hline
\end{tabular}

Fuente: elaboración propia usando datos del Political Database of the Americas, Georgetown University

Conforme a lo dispuesto en la Constitución, cinco años más tarde, llegó el momento de renovación de los mandatos de los diputados. En 4 
de diciembre de 2005, se llevaron a cabo nuevas elecciones parlamentarias en Venezuela. No obstante los principales partidos de oposición (Acción Democrática, COPEI Primero Justicia y Proyecto Venezuela) decidieron retirarse de la contienda alegando falta de confianza en el Consejo Nacional Electoral. El resultado se puede ver en la siguiente tabla.

Tabla 6: Elecciones Parlamentarias de 2005 en Venezuela

\begin{tabular}{c|c}
\hline \multicolumn{2}{c}{ Tabla 6: Elecciones Parlamentarias de 2005 en Venezuela } \\
\hline Partido & Escaños \\
\hline MVR & 114 \\
\hline Podemos & 15 \\
\hline PPT & 11 \\
\hline MEP & 11 \\
\hline PCV & 8 \\
\hline UPV & 8 \\
\hline Total & 167 \\
\hline
\end{tabular}

Fuente: elaboración propia usando datos del Political Database of the Americas, Georgetown University.

Como se observa en la tabla anterior, frente al absentismo de la oposición, el chavismo logró casi todos los escaños en la Asamblea Nacional, lo que hizo posible la aprobación de la Ley Habilitante propuesta por Chávez en 2007-2008. En la coyuntura, el oficialismo fue acusado de utilizar dicho mecanismo para aprobar la reforma rechazada por la población en el referendo constitucional de 2007, cuya finalidad era modificar 69 artículos de la Constitución de 1999, entre los cuales estaba el de conformar a Venezuela como Estado socialista. Aunque no sea de todo correcto, entre los decretos aprobados está la Ley para el Fomento y Desarrollo de la Economía Popular que puede ser vista como un primer paso en la conversión de Venezuela hacia al Estado Comunal, proclamado en 2010, también por Ley Habilitante.

La hegemonía chavista en la Asamblea, también permitió la aprobación de la Ley Orgánica de Procesos Electorales, aprobada el 31 de julio de 2009. Su contenido regula los principios constitucionales en los procesos electorales, que establece en el artículo 63 el "sistema electoral paralelo", definiéndolo como sistema electoral mixto de personalización del sufragio para los cargos nominales (electos por mayoría de votos) y de representación proporcional para los cargos por lista, en el cual la elección nominal no incide en la elección proporcional mediante lista.

En la votación proporcional de los diputados en lista, que representan un total de 51 escaños, se reparten en cada una de las entidades federales una cuota que varía entre dos y tres escaños, conforme su población ${ }^{8}$. En la votación mayoritaria los ciudadanos escogen directamente el nombre de 113 diputados elegidos en 87 circunscripciones electorales (municipios y parroquias), que se dividen en uninominales y plurinominales, en las primeras los ciudadanos eligen un solo diputado, el las segundas es posible elegir a más de un (dos o tres). Además, la Ley Orgánica de Procesos Electorales establece un sistema de mayoría relativa, en circunscripciones especiales uninominales por región, para el sufragio de tres diputados por la representación indígena.
8. Este criterio ha dado lugar a considerables críticas de la oposición, puesto que los estados menos poblados (donde Chávez tiene una mayoría, ya que también son los más pobres) están sobrerrepresentados. 
La primera elección celebrada bajo las nuevas reglas fueron las Parlamentarias de 2010, que también marcaron el debut de coalición opositora Mesa de Unidad Democrática (MUD), derrotada por el oficialismo, ahora reunido en el PSVU, que obtuvo 98 de los 165 escaños, frente a 65 de la MUD. Sin embargo, como se observa en la tabla abajo el chavismo no logró obtener la mayoría absoluta de 99 diputados (3/5 partes) requerida para la aprobación de las leyes habilitantes.

Tabla 7: Elecciones Parlamentarias de 2010 en Venezuela

\begin{tabular}{c|c}
\hline Partido & Porcentaje de votos \\
\hline PSUV & $45,80 \%$ \\
\hline PJ & $9,50 \%$ \\
\hline AD & $8,00 \%$ \\
\hline Un Nuevo Tiempo & $7,00 \%$ \\
\hline COPEI & $4,40 \%$ \\
\hline PPT & $4,10 \%$ \\
\hline PV & $3,50 \%$ \\
\hline Por la Democracia & $2,90 \%$ \\
\hline \multicolumn{2}{c}{ Distribución de Escaños } \\
\hline \multicolumn{2}{c}{ PSUV } \\
\hline MUD (coalición oposicionista)
\end{tabular}

Fuente: elaboración propia usando datos del Political Database of the Americas, Georgetown University

El 22 de junio de 2015, la presidenta del Consejo Nacional Electoral (CNE), Tibisay Lucena, estableció la fecha para la realización de las elecciones parlamentarias de Venezuela de 2015, que se celebrarán el 6 de diciembre de 2015. En un artículo reciente Steve Ellner (2015), reiteró la importancia de dicha contienda para la definición del futuro del proceso de cambio en Venezuela, cuya continuidad se debe a sorprendentes éxitos electorales obtenidos en circunstancias a veces francamente desfavorables. Citando una declaración dada en mayo por, Jesús Torrealba, jefe de MUD, Ellner sostiene que la coalición espera ganar el control de la Asamblea para derrocar a Maduro, adoptando una estrategia llamada por los chavistas de "opción paraguaya" en referencia a la destitución del presidente Fernando Lugo por decisión del congreso en 2012 (HERNÁNDEZ, 2015).

La misma maniobra se formuló durante las elecciones a nivel municipal celebradas en diciembre de 2013, presentada por los líderes oposicionistas como un "plebiscito" para determinar la continuidad de Maduro en el poder. No obstante, cuando los chavistas emergieron victoriosos con un margen de diferencia de 11,5 puntos porcentuales, la oposición nuevamente abandona la estrategia legalista y se vuelve a agitaciones insurreccionales. Ahora capitaneada por el movimiento La Salida, los oposicionistas siguen en un patrón de conducta que caracteriza su papel subordinado en la lucha hegemónica en Venezuela, una vez que la variación sus estrategias todavía ha sido condicionada por los éxitos electorales del chavismo. 
Conclusiones

Los factores presentados a lo largo de estas páginas nos sugieren puntos de aproximación y de distanciamiento en los contextos políticos boliviano y venezolano, especialmente cuanto a la reconfiguración de la oposición y las posibles implicaciones en el Legislativo. Como señalamos anteriormente, la oposición en Bolivia se constituye de manera más plural en la medida en que está conformada no sólo por la élite política oligárquica contraria al cambio - compuesta por empresarios y agroextractivistas - sino también por actores disidentes al gobierno de Evo Morales, representados por ex miembros del oficialismo y grupos indígenas. En este punto, observamos una clara distinción entre ser oposición al proceso de cambio y ser contrario al gobierno que, en el caso de los primeros, se sobrepone. Sin embargo, lo mismo no ocurre en relación a los disidentes, que se ven contrarios al MAS y a la captura del proceso por el gobierno, y no al cambio en sí. En ese sentido, reivindican que los indígenas retomen su posición central, independiente del partido gubernista, y recuperen el ethos descolonizador que se perdió frente a las medidas autoritarias promocionadas por el Ejecutivo.

Mientras en Bolivia la renovación del antagonismo fue acompañada por la pluralización dentro de la oposición al gobierno, en Venezuela en escenario político se muestra menos plural. Aunque la radicalización de los embates entre la oposición y la gestión de Maduro haya dado paso al surgimiento de la disidencia, a ejemplo de la creación de Marea Socialista, esta se muestra todavía incipiente y sin representación parlamentaria. Así mismo, da diferencia entre "dialogadores" y "salistas" se diluye en su posición contraria no solamente al gobernó sino también al proceso de cambio. En ese sentido, las relaciones de fuerzas en Venezuela siguen centradas en la disputa entre dos bloques, enyesada y articulada alrededor del chavismo. En lo que respecta al Legislativo, las elecciones de 2015 pueden imponer cambios significativos en la lucha hegemónica trabada en el país, sobretodo, en caso de derrota del bloque oficialista que, desde 1999, gobierna con el pleno apoyo del Legislativo. La Asamblea, por supuesto, se queda reducida a mera instancia de aclamación de las decisiones del Executivo y por lo tanto incapaz de desempeñar su papel en la formación de consensos y representación de minorías parlamentarias.

Con relación a Bolivia, la mayoría lograda por el MAS en las elecciones de 2009 y 2014 garantizó al oficialismo la aprobación de sus proyectos en el Congreso. Pero si este escenario sugiere la pérdida de relevancia de los procesos de negociación entre los grupos parlamentarios, lo mismo no se puede afirmar respecto a las relaciones entre el gobierno y la sociedad civil, a ejemplo de las movilizaciones en defensa del TIPNIS. Organizadas por la Cidob, las marchas contaron con el apoyo de la Conamaq además de una comunidad epistémica y los desilusionados con el proceso de cambio. También el "gasolinazo" pone en duda la hegemonía gubernista. Por fin, el éxito oficialista es puesto en jaque por la emergencia de nuevas articulaciones de los disidentes y su posición en puestos importantes en el nivel sub nacional, antes ocupados por masistas. 
Referencias

ARDITI, B. Populism as an Internal Periphery of Democratic Politics. In PANIZZA, Francisco (Ed.). Populism and the Mirror of Democracy. London/New York: Verso, 2005, p. 73-104.

ACOSTA, A. Extractivismo y neoextractivismo: Dos caras de la maldición. In: LANG, M. y MOKRANI, D. (compiladoras). Más allá del desarrollo. Quito: Fundación Rosa Luxemburg/ Abya-Yala, 2011.

ALEXANDRE, C. Bolívia: instabilidade política e dificuldade de inserção regional. In: LIMA, M. R. S. de e COUTINHO, M. V. (orgs.). A Agenda Sul-Americana: Mudanças e Desafios no Início do Século XXI. Brasília: Fundação Alexandre de Gusmão, 2007.

ALMARAZ, A. et al. Manifiesto 22 de Junio de 2011. Por la recuperación del proceso de cambio para el pueblo y con el pueblo. Disponible en: $<$ http://www.herramienta.com.ar/herramienta-web-9/bolivia-manifiesto-22-de-junio>. Accedido en: 20 oct. 2015.

ARIÑEZ, R. 2015. Evo anuncia que la carretera que atraviesa el TIPNIS `se realiza`. La Razón, 05.06.2015. Disponible en: <www.la-razon.com/nacional/Evo-carretera-atraviesa-TIPNIS-realiza_0_228397>. Accedido en: 05 jun. 2015.

AZCUI, M. 2014. El Tribunal Electoral de Bolivia corrige la nueva distribución de escaños. El País, 15.05.2014. Disponible en: < http://internacional.elpais.com/internacional/2014/05/15/actualidad/1400105632_993470.html>. Accedido en: 20 jun. 2015.

BRESSER-PEREIRA, Luiz Carlos. Do antigo ao novo desenvolvimentismo na América Latina. Texto para Discussão, n. 274, 2010

BOLIVIA. Ley N.3364. Ley Especial de Convocatoria a la Asamblea Constituyente. La Paz, 6 de marzo de 2006. Ley N.3365. Ley de Convocatoria al Referéndum Nacional Vinculante a la Asamblea Constituyente para las Autonomías Departamentales. La Paz, 6 de marzo de 2006. Ley N. 4.021. Ley del Régimen Electoral Transitorio. La Paz, 14 de abril de 2009. Nueva Constitución Política del Estado. Vicepresidencia de la República, Presidencia del Honorable Congreso Nacional de Bolivia, 2008.

CAMPELLO, D. The politics of financial booms and crises: Evidence from Latin America. Comparative Political Studies, n.47.2, 2014.

CLAROS, H. 2012. Se inaugura Escuela de Formación Política del MAS-IPSP. Agencia Plurinacional de Comunicación, 30.05.2012. Disponible en: <http://www.apcbolivia.org/inf/noticia. aspx $?$ fill $=950 \& \mathrm{Id}=8 \& \mathrm{D} 86 \mathrm{Fv} \&$ fil $=9 \&$ hsdate $=10 \& \mathrm{BD} 54 \mathrm{SSDfecrada}=\& \% \mathrm{FS} 45>$. Accedido en: 28 jul. 2015.

DELGADO, A. C. T. Guerreiros do Arco-Íris: os caminhos e descaminhos da descolonização na Bolívia no início do século XXI. Rio de Janeiro. Tese (Doutorado, em Relações Internacionais). Pontifícia Universidade Católica do Rio de Janeiro, 2014.

The TIPNIS Conflict in Bolivia. Contexto Internacional, vol. 39(2) May/Aug 2017.

ELLNER, S. El dilema populista, el pragmatismo y la izquierda en el poder en Venezuela. Rebelión, 02.06.2015. Disponible en: http://www.rebelion.org/noticia.php?id=199495 . Accedido en: 18 abr. 2015.

EL IMPULSO. Barquisimeto, Venezuela. Disponible en: <http://www.elimpulso.com/>. Accedido en: jul. 2015.

EL UNIVERSAL. Caracas. Disponible en: <http://www.eluniversal.com/localizadores/ elecciones2015?p=ELECCIONES\%202015>. Accedido en: 17 mar. 2015.

EL NACIONAL. "Eligen delegados para el III Congreso del Psuv". El Nacional WEB, 20.07.2014. Disponible en: <http://www.el-nacional.com/politica/Eligen-delegados-III-Congreso-Psuv_0_449355195.html>. Accedido en: 30 jan. 2015.

FRENCH-DAVIS, R. Macroeconomía, comercio y finanzas para reformar las reformas en América Latina. Chile: McGraw-Hill, Cepal, 1999.

GARCÍA, X. 2013. Las Organizaciones Sociales Venezolanas como espacio de actuación del Trabajo Social. Caso Consejos Comunales. CONSIDERACIONES TEORICAS e INTERVENCION DEL TRABAJO SOCIAL. RUMBOS TS, año VII (8), p.59-71.

GOULART, M. ¿La Calle es la salida? Uma hipótese sobre o 12F Venezuelano. In: Breviário de Filosofia Pública, v. 119, n. 20, p.24-36, 2014.

GUDYNAS, E. Diez tesis urgentes sobre el nuevo extractivismo. Contextos y demandas bajo el progresismo sudamericano actual. In: GUDYNAS, E. et al. Extractivismo, política y sociedad. 
Quito: CAAP (Centro Andino de Acción Popular) y CLAES (Centro Latino Americano de Ecología Social), 2009.

HERNÁNDEZ, C. Una asamblea para derrocar a Maduro. El Universal, 09.05.2015. Disponible en: <http://www.eluniversal.com/noticias/opinion/una-asamblea-para-derrocar-maduro_37609>. Accedido en: 20 set. 2015.

LACLAU, E. On Populist Reason. London: Verso, 2005.

LACLAU, E e MOUFFE, C. Hegemonía y estrategia socialista. Madrid: Siglo Veintiuno de España, 1987.

LÓPEZ MAYA, M. Venezuela 2001-2004: actores y estrategias en la lucha hegemónica. In: CAETANO, G. (ed.). Sujetos sociales y nuevas formas de protesta en la historia reciente de América Latina. Buenos Aires: CLACSO, 2006.

MOLINA, F. Balotajes confirman que "el evismo es mucho más fuerte que el masismo". Infolatam, 4 de mayo de 2015. Disponible en: <http://www.infolatam.com/2015/05/04/balotajes-confirman-queel-evismo-es-mucho-mas-fuerte-que-el-masismo/>. Accedido en: 06 mai. 2015.

OIT. Convênio 169 da Organização Internacional do Trabalho (OIT) sobre Povos Indígenas e Tribais.Escritório Brasil. 2011.

PATZI, F. Ilusiones y Desilusiones del "proceso de cambio". Seguimiento cotidiano de los principales conflictos en el Gobierno del Movimiento al Socialismo 2006-2012. La Paz: "All Press" Labores Gráficas, 2013.

MODElo COMUNAL. Propuesta Alternativa para Salir del Capitalismo y del Socialismo. La Paz: All Press, 2013a.

PIZARRO, R. La vulnerabilidad social y sus desafíos: una mirada desde América Latina. Estudios Estadísticos y Prospectivos. Comisión Económica para América Latina y Caribe. Santiago de Chile: Naciones Unidas, 2001.

PRADA ALCOREZA, R. Descolonización y transición. Quito: Ediciones Abya-Yala, 2014.

POLITICAL DATABASE OF THE AMERICAS, Georgetown University. Disponible en: $<$ http://pdba.georgetown.edu/Elecdata/elecdata.html $>$. Accedido en: jul. 2015.

SALAMANCA, L. Mapa social y político de los sectores populares. Revista SIC, Caracas, n.738, p. 1-41, oct. 2011.

SVAMPA, M. “'Consenso de los Commodities” y lenguajes de valoración en América Latin', Nueva Sociedad (244): 30-46, 2013.

TAPIA, L. El estado de derecho como tiranía. La Paz: CIDES-UMSA y Autodeterminación, 2011.

TSE. Resultados de las Elecciones Generales 2014. Disponible en: <http://www.oep.org.bo/ index.php/procesos-electorales/elecciones-generales-2014/798-resultados-de-las-elecciones-generales-2014>. Accedido en: 15 jun. 2015.

TSE. Referendum Constitucional 2016. Resultados Finales. Disponible en: <http://www. oep.org.bo/index.php/procesos-electorales/referendo-constitucional-2016> . Accedido en: 15 jun. 2015.

VELTMEYER, H. and PETRAS, James. A Post-Neoliberal Development Model or Imperialism of the Twenty-First Century? London: Zed Books,2014

VENEZUELA. Constitución de la República de Venezuela. Disposiciones Transitorias. Gaceta Oficial de la República de Venezuela: No. 662, extraordinaria, 23 de enero de 1961. Constitución de la República Bolivariana de Venezuela. Gaceta Oficial de la República de Venezuela. Año CXXVII-Mes III. Caracas, jueves 30 de diciembre de 1999 